\title{
Production and characterization of large-size diamond detectors for particle tracking and medical applications
}

\section{J. Collot ${ }^{1}$, A. Bes, G. Bosson, S. Curtoni, D. Dauvergne, L. Gallin-Martel, M.-L. Gallin-Martel, J.-F. Muraz, J. Hostachy, A. Lacoste, S. Marcatili, F. Rarbi,} M. Yamouni

LPSC, université Grenoble Alpes, CNRS/IN2P3

53 avenue des Martyrs, 38026 Grenoble cedex 9 France

\section{J.-M. Brom}

IPHC, université de Strasbourg, CNRS/IN2P3

23 rue du loess - BP 28 - 67037 Strasbourg cedex 2 France

\section{J. Morse, W. De Nolf, M. Salomé}

ESRF

71 avenue des Martyrs, CS 40220, 38043 Grenoble cedex 9, France.

\section{J. Krimmer, É. Testa}

IPNL, université de Lyon, CNRS/IN2P3

Domaine scientifique de la Doua, bâtiment Paul Dirac, 4 rue Enrico Fermi, 69622 Villeurbanne cedex, France

Large-size diamond detectors have been produced and tested with the aim of achieving reliable \& efficient sensors for particle tracking or medical applications. Poly- and single-crystal CVD diamond samples were submitted to various ionizing particles. Their metallization was performed by using Distributed MicroWave Plasmas, a process developed by LPSC. Their detection performance was investigated using $\alpha$ and $\beta$ radioactive sources, $95 \mathrm{MeV} / \mathrm{u}$ carbon beams from GANIL (Caen France) and short-bunched $8.5 \mathrm{keV}$ photons from ESRF. This study is part of the ANR project MONODIAM-HE and of the CLaRyS collaboration for the on-line dose monitoring of hadrontherapy. The goal here is to provide large-area detectors with a high detection efficiency for carbon or proton beams, yielding time and position measurement at count rates greater than $100 \mathrm{MHz}$. A time resolution ranging from 20 ps up to $40 \mathrm{ps}$ and an energy resolution varying from $7 \%$ up to $10 \%$ were measured. It allowed us to conclude that poly-crystal CVD diamond detectors are good candidates for this application.

The European Physical Society Conference on High Energy Physics

5-12 July, 2017

Venice

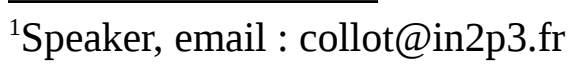




\section{Motivation}

The treatment of tumors by a beam of carbon ions or protons is an alternative radiotherapy treatment of cancer known as hadrontherapy. Hadrontherapy allows a more efficient dose delivery in tumors, while reducing the dose deposited in the nearby healthy organs. The conformation of the deposited dose to tumors is provided by distributing Bragg peaks in their volume, either spot by spot, or by means of passive scattering and energy spreading. However, since multiple sources of uncertainty on the ion path may cause deviations from the planned dose distribution [1], online control of the ion path is desired in order to reduce safety margins and optimize the ballistic advantage of hadrontherapy.

During an irradiation with ion beams, nuclear reactions occur for part of the projectiles along their path in the patient body, and photons in the 1-10 MeV range are emitted almost isotropically within much less than a picosecond. It has been experimentally shown that the longitudinal distribution of such prompt-gamma production is highly correlated in space to the primary ion path [2], [3]. In the CLaRyS collaboration, two systems for prompt gamma detection are currently under development [4], [5], [6]: a collimated gamma camera and a Compton camera. The originality of the two systems is the use of Time-of-Flight (TOF) in order to reduce the background. Depending on the beam time-structure and intensity, a beam tagging hodoscope may be necessary to detect the arrival time of ion bunches or even individual projectiles. This hodoscope will also be used for transverse position measurement, giving access to $3 \mathrm{D}$ prompt-gamma imaging. Thus the hodoscope plays a major role in all the detection concepts, and has to overcome the issue of providing position and timing information of the incident hadron beam at about $100 \mathrm{MHz}$ count rates. The collaboration has already developed a scintillating-fiber based hodoscope, read-out by photomultipliers [6], [7]. In order to improve the timing resolution, and to overcome the count rate limitation of photomultipliers, the collaboration initiated the MoniDiam project that aims to develop a diamond-based hodoscope and its dedicated integrated fast read-out electronics.

\section{Material \& methods}

\subsection{Diamond sensors}

Chemical Vapor Deposition (CVD) synthetic diamonds are grown by the technique of microwave plasma enhanced CVD. By tightly controlling their growth parameters, their qualities and detection properties may be made reproducible. For the homo-epitaxial ones, diamond is used as a seed for the CVD growth. Diamond nano-crystals are used as substrate for the pCVD (poly-crystal CVD). The resulting diamond bulk contains grain-boundaries corresponding to the junction of crystal growth from different seeds. These structural defects act as free charge carrier trapping centers. The main asset of pCVD diamonds is that they can be grown with large areas of several $\mathrm{cm}^{2}$ [8]. Higher quality CVD diamond can be obtained by using HPHT (High Pressure High Temperature) diamond seeds to grow single crystals (sCVD). However, given the availability of the HPHT seeds, the area of these diamonds remains, up to now, less than $1 \mathrm{~cm}^{2}$. Last, hetero-epitaxial diamonds are grown using CVD on a substrate featuring a diamond-like crystal structure, for example iridium (hereafter named as Diamond- 
on-Iridium, DOI). These diamonds feature qualities close to those of sCVD crystal. Moreover, they can be grown above $1 \mathrm{~cm}^{2}$ in area [9], [10].

Compared to other semiconductor detectors (e.g. Si detectors) CVD diamond sensors exhibit several advantages [11], [12], [13], [14]. They have been demonstrated to be radiation hard. They feature a high resistivity $\left(>10^{13} \Omega \mathrm{m}\right.$ ) coupled to a large electronic gap (5.48 eV). This results in a lower noise level and an almost negligible leakage current even at room temperature. Despite the fact that the energy required to produce electron-hole pairs is 3.6 times larger than in silicon, the signal-to-noise ratio is higher for diamond. The high charge carrier mobility leads to a very fast detector response allowing excellent time resolution (few tens of picoseconds) and high count rate capability, even for polycrystalline detectors.

Hence, diamond applications are more and more considered in various physics fields. Diamond detectors are commonly used as X-ray synchrotron radiation monitors but also as UV and neutron sensors [15]. In high-energy physics, diamond detectors are used for beam monitoring [16]. In medical physics applications, they are used as dosimeters for radiotherapy [17].

In the present study, we used sCVD and pCVD diamonds from Element6 [8], and heteroepitaxial DOI diamonds from Audiatec [9]. The tested samples had dimensions ranging from $300 \mu \mathrm{m}$ up to $500 \mu \mathrm{m}$ in thickness, and from $5 \times 5 \mathrm{~mm}^{2}$ to $2 \times 2 \mathrm{~cm}^{2}$ in area.

\subsection{Detector processing and signal readout}

An aluminum disk shaped metallization was locally performed by using the DMW (Distributed Microwave Plasmas), a technology developed by LPSC [18]. The crystal surface preparation and metal deposition were thus performed by a sequential plasma process consisting in two steps of reactive plasma cleaning, followed by plasma-assisted sputtering.

The sensor contact for the disk-shaped metallization consists of a $50 \mathrm{~nm}$ thick aluminum layer deposited on both sides. Diamonds are mounted on sample holders with $50 \Omega$ adapted impedance and SMA connectors, enabling reversible bias and signal readout from both sides of the diamond. Several preamplifiers were tested : the CIVIDEC C2 low-noise broadband RF amplifier (from CIVIDEC Instrumentation company [19]) and the DBA III (Diamond Broadband Amplifier [20]) from GSI. These amplifiers are intended for use with fast detectors. They exhibit a $50 \Omega$ input impedance and are able to work with FWHM pulse widths of less than $1 \mathrm{~ns}$.

\subsection{Waveform readout}

The signal waveform readout was performed with a $500 \mathrm{MHz}, 3.2 \mathrm{GS} / \mathrm{s}$ digital sampling 'Wavecatcher' [21] system. This system could be configured in a continuous acquisition mode, recording a set of waveforms, and thus enabling large statistics offline analysis.

In addition at ESRF, a $2 \mathrm{GHz}, 20 \mathrm{GS} / \mathrm{s}$ analog bandwidth DSO (LeCroy 620Zi) [22] was used. The DSO data acquisition was fully integrated into the ESRF ID21 SPEC software acquisition framework which enabled us to measure the temporal response of the detectors as they were scanned in the beam, thus creating $2 \mathrm{D}$ response maps of the detector surfaces. 


\section{Characterization}

\subsection{Measurement with $\alpha$ and $\beta$ particles}

Two dedicated test benches, with radioactive $\alpha$ and $\beta$ sources $\left({ }^{233} \mathrm{U},{ }^{241} \mathrm{Am},{ }^{90} \mathrm{Sr}\right)$ were used. The mean free path of $\alpha$ particles (about $12 \mu \mathrm{m}$ ) is much smaller than the diamond thickness. In this case, all the current due to the drift of charge carriers comes from a single species (either electrons or holes, depending on the bias polarity) traversing the whole detector thickness. This enabled us to record the signal shape as a function of the electric field and as a function of the diamond side (growth or substrate sides) where the initial charges were produced.

On the contrary, the $\beta$ setup enabled us to infer the Charge Collection Distance (CCD) since the high-energy electrons emitted by the ${ }^{90} \mathrm{Sr}$ source traverse the diamond (a trigger on transmitted high-energy electrons is used). As an illustration, a measurement done with a $0.45 \mathrm{x}$ $0.45 \mathrm{~cm}^{2}$ x $518 \mu \mathrm{m}$ sCVD diamond manufactured by Element 6 is shown on Fig. 1. The asymptotic value obtained for the CCD by increasing the electric field applied on diamond electrodes is very close to the diamond thickness, and corresponds to full collection of the ionized charge. This illustrates the quality of the SCVD diamond.

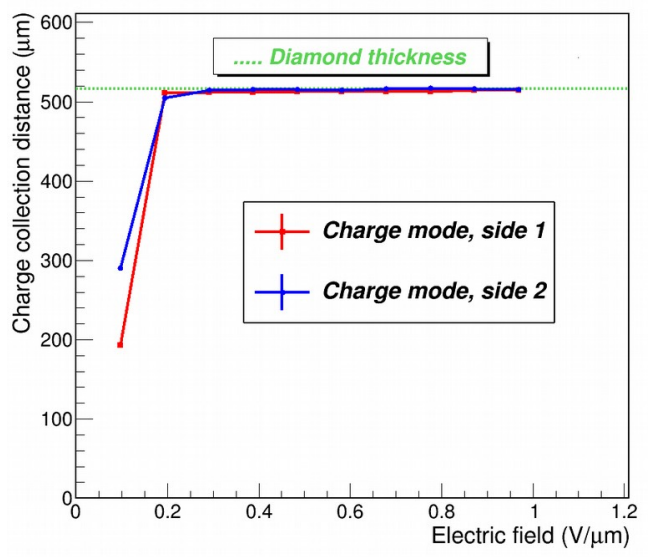

Fig. 1. Charge collection distance measured on a 0.45 x $0.45 \mathrm{~cm}^{2}$ x $518 \mu \mathrm{m}$ sCVD diamond manufactured by Element6. As shown by the green line, beyond $0.3 \mathrm{~V} / \mu \mathrm{m}$, the charge collection is complete. Sides 1 and 2 refer to the growth and substrate sides.

\subsection{Measurement with X-ray beams}

XBIC (X Beam Induced Current) is of huge interest for testing diamond. Here, a $8.5 \mathrm{keV}$ photon focused \& bunched micro-beam with a well-defined time structure was used at ESRF. As regards energy deposition in the diamond, $35 \%$ of the beam is absorbed in a $300 \mu \mathrm{m}$ thick diamond, which makes the energy deposit almost constant and continuous along the beam path (similar to a high-energy electron). In the ESRF 4-bunch mode, the $\sim 100$ ps duration X-ray pulses, containing a fixed number of photons, adjustable by means of attenuators, with a maximum value of $\sim 1400$, mimic the passage of single homogeneously-ionizing particles 
(maximum energy deposit $\sim 4.1 \mathrm{MeV}$ ). Photon bunches are separated by 704 ns intervals, which makes it possible to record individual pulses, as well as continuous current integration with picoammeters.

The diamond samples were placed inside an electromagnetic shielding box. The box was positioned with micrometric reproducibility at the sample position of the micro-diffraction end station (in air) of the ID21 beamline at ESRF.

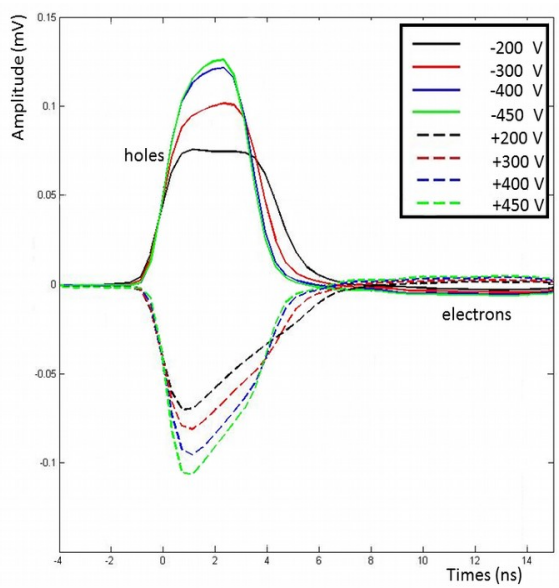

Fig 2. Signal measurement with an $\alpha$ source on a $0.5 \times 0.5 \mathrm{~cm} 2 \times 300 \mu \mathrm{m}$ DOI detector.

\subsection{Measurements with a $95 \mathrm{MeV} / \mathrm{u} 12 \mathrm{C}$ ion beam}

Finally, to evaluate the diamond performance under carbon ion irradiation, a test on a $95 \mathrm{MeV} / \mathrm{u}{ }^{12} \mathrm{C}$ ion beam was carried out at the GANIL facility in Caen (France). The energy deposited by such ions in a $300 \mu \mathrm{m}$ thick diamond is $25 \mathrm{MeV}$. This experimental setup enabled us to evaluate single carbon ion detection characteristics.

\section{Results}

In the following sections, we report on the charge collection properties of CVD diamonds [23] with disk-shaped metallized sensors.

\subsection{Pulse shape analysis}

Diamond detectors allow particle identification by pulse-shape analysis. The pulse-shape of the signal collected in diamond material is determined by i) the ionization profile in the diamond sensor, which depends on the particle type and energy, and ii) the transport properties of charge carriers, which depend on the dimensions and properties of the diamond (bias, presence of defects). For very pure crystals such as sCVD diamonds, the expected shape of the current pulse due to an $\alpha$ particle is rectangular, since it results from the drift of a constant amount of charge carriers created close to the surface along the whole crystal thickness. If space charges or trapping defects are present in the diamond bulk, such as for pCVD diamond, the rectangular shape would rather be rhombic. On Fig. 2, the signals recorded on heteroepitaxial diamonds grown on iridium for holes and electrons carriers are plotted by varying the diamond 
bias voltage using the $\alpha$ test bench. It appears that such diamonds behave as SCVD crystal for the hole carriers and as PCVD for the electron ones. This particular behavior of DOI detector signals was already reported [24].

\subsection{Time resolution measurements}

Diamond detectors are expected to be fast responding detectors where rise times smaller than $1 \mathrm{~ns}$ are observed. Consequently, an offline procedure was established to determine the potential time resolution of the detectors, based on the waveform analysis. A numerical (offline) Constant Fraction Discrimination (CFD) was performed by averaging the background, determining the maximum of the pulses, and interpolating the $50 \%$ rise time value. At the GANIL facility, the dedicated experimental setup enabled us to measure a time resolution between two diamond samples positioned one behind the other. In Fig. 3, a time resolution lower than $18 \mathrm{ps}$ rms was reached using a $0.45 \times 0.45 \mathrm{~cm}^{2} \times 518 \mu \mathrm{m}$ single-crystal sample provided by Element 6 coupled to a $0.5 \times 0.5 \mathrm{~cm}^{2} \times 300 \mu \mathrm{m}$ DOI provided by Audiatec. The DOI detector was read out with a CIVIDEC preamplifier, and the sCVD with a DBAIII preamplifier, with smaller gain. Thus, this time resolution value results from the quadratic sum of both detection device signals. Such results with single readout channels are very promising, but, in view of the developpment of a multichannel detector prototype, integrated very fast front-end electronics will be needed.

Tests were also carried out at the GANIL facility with a $2 \times 2 \mathrm{~cm}^{2} \times 500 \mu \mathrm{m}$ sample coupled to a $1 \times 1 \mathrm{~cm}^{2} \times 500 \mu \mathrm{m}$ one. Both diamonds were pCVDs provided by Element 6 . There, the time resolution was measured at the level of $90 \mathrm{ps}$. This result is not in contradiction with our previous observation, since the active areas and hence the detector capacitance which are here about one order of magnitude larger, impact the time resolution [14].

\subsection{Energy resolution measurements}

Energy resolution measurements (Fig. 4) were performed both at ESRF and GANIL. At GANIL, with a $95 \mathrm{MeV} / \mathrm{u}$ carbon ion beam impacting a $0.5 \times 0.5 \mathrm{~cm}^{2} \times 300 \mu \mathrm{m}$ polycrystalline diamond, an energy resolution of $\sim 7 \%$ rms was reached. Using the same diamond sensor, with the $8.5 \mathrm{keV}$ pulsed photon beam at ESRF, an energy resolution of $\sim 9 \%$ rms was obtained. Such resolutions are good enough to identify unambiguously the passage of a single ion.

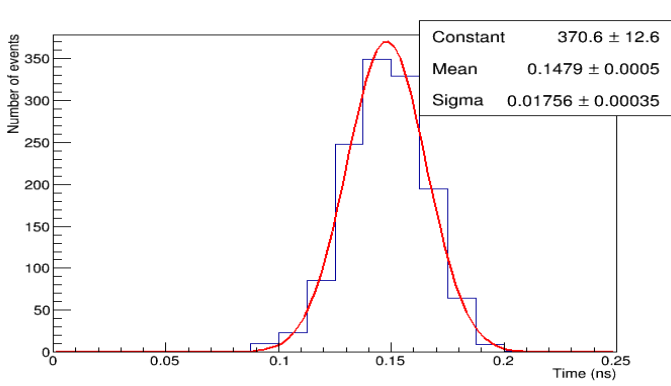

Fig. 3 Time resolution measured between a $0.45 \times 0.45 \mathrm{~cm}^{2} \times 518 \mu \mathrm{m}$ sCVD diamond and a $0.5 \times 0.5 \mathrm{~cm}^{2} \times 300 \mu \mathrm{m}$ DOI detector, traversed by a single carbon ion at $95 \mathrm{MeV} / \mathrm{u}$ incidence energy.

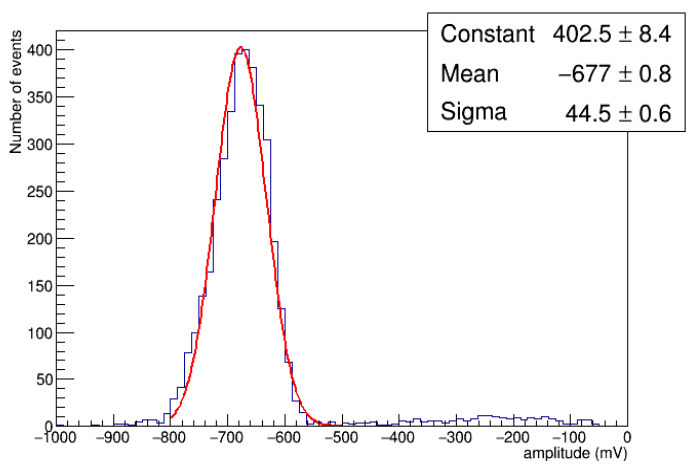

Fig. 4 Energy resolution measured on a $0.5 \times 0.5 \mathrm{~cm}^{2} \times 300 \mu \mathrm{m}$ poly-crystalline diamond with $95 \mathrm{MeV} / \mathrm{u}$ carbon ion beam. Here, the mean energy deposit is $25 \mathrm{MeV}$. 


\subsection{Surface scanning}

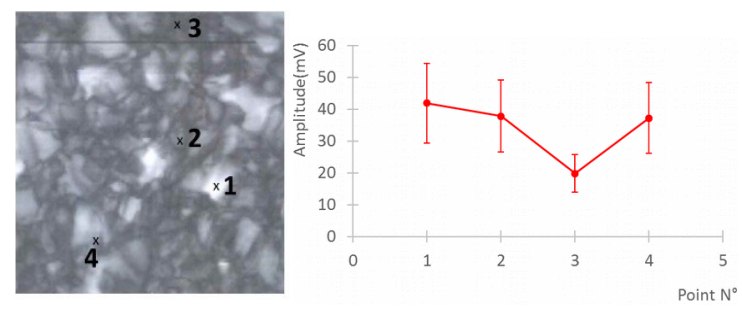

Fig. 5 Amplitude (right) of the signal versus the ESRF-ID21 pencil beam localization from point 1 up to 4 on the current-readout surface map (left) of a $1 \mathrm{~mm} 2$ area of a $1 \times 1 \mathrm{~cm} 2 \times 500 \mu \mathrm{m}$ polycrystalline diamond detector.

At ESRF, we were able to record maps of the signal response in current integration mode as a function of the $\mathrm{x}, \mathrm{y}$ beam position. Fig. 5 shows an example of a polycrystalline detector map $\left(1 \times 1 \mathrm{~cm}^{2} \times 500 \mu \mathrm{m}\right.$ pCVD detector from Element 6$)$ on a $1 \mathrm{~mm}^{2}$ area. The grey scale corresponds to the charge collection efficiency measured by an electrometer. Clearly, the response of the detector reflects the spatial distribution of grain boundaries in the polycrystalline material.

In addition, Fig. 5 shows the diamond response versus the photon pencil beam localization for the same pCVD diamond detector. With such a sample, a difference of a factor two is observed between the clearest point and the darkest one. It has been checked that such an amplitude variation will introduce an acceptable walk in time measurement. This result could be seen as in contradiction with the result of the previous section, where a resolution of $9 \%$ was obtained with a similar pCVD. Nevertheless, one has to note that the present sample is different $\left(1 \mathrm{~cm}^{2}\right)$ and that we measured the difference between the two extreme response regions of the surface.

\section{Conclusion}

Diamond detectors are being studied in Grenoble for multiple applications in nuclear physics, in high-energy physics and for medical applications. In particular, large-area, synthetic pCVD or DOI diamond detectors are investigated for on-line hadrontherapy beam tagging applications. In this context, they will be used as a ultra-fast hodoscope associated to a gamma camera as proposed by the CLaRyS collaboration. Their radiation hardness, very fast response and good signal-to-noise ratio make them good candidates for this type of applications.

We carried out tests with disk-shaped metallized diamonds. A time resolution ranging from 20 up to 90 ps rms, and an energy resolution ranging from $7 \%$ up to $9 \%$ rms were measured, when irradiating the whole surface of CVD diamonds with various ionizing particles. These results satisfy our requirements despite the obvious non-uniformity of the crystalline structure observed thanks to XBIC analyses. 


\section{Acknowledgement}

The authors would like to acknowledge the ESRF-ID21 beamline for provision of synchrotron radiation with experiments MI-1243 (2016) and MI-1285 (2017). This work was supported by the Labex PRIMES (ANR-11-LABX-0063), FranceHadron (ANR-11-INBS-0007) and ANR MONODIAM-HE (ANR-089520). The authors are grateful to Matthias Schreck and Martin Fischer from Audiatec (Augsburg) for providing the LPSC laboratory with DOI samples. Dominique Breton, Jihanne Maalmi from LAL-Orsay and Eric Delagnes from CEA Saclay are thanked for their implication in dedicated software development and technical support of the Wavecatcher data acquisition system.

\section{References}

[1] H. Paganetti, Range uncertainties in proton therapy and the role of Monte Carlo simulations, Phys. Med. Biol., vol. 57, no. 11, pp. R99-R117, June 2012.

[2] C.-H. Min, C. H. Kim, M.-Y. Youn, and J.-W. Kim, Prompt gamma measurements for locating the dose falloff region in the proton therapy, Appl. Phys. Lett., vol. 89, no. 18, pp. 183517-3, Oct. 2006.

[3] E. Testa et al., Monitoring the Bragg peak location of $73 \mathrm{MeV} / 4$ carbon ions by means of prompt $\gamma$ ray measurements, Appl. Phys. Lett., vol. 93, no. 9, p. 93506, 2008.

[4] J. Krimmer et al., Collimated prompt gamma TOF measurements with multi-slit multi-detector configurations, J. Instrum., vol. 10, no. 1, pp. P01011-P01011, Jan. 2015.

[5] J. Krimmer et al., Development of a Compton camera for medical applications based on silicon strip and scintillation detectors, Nucl. Instrum. Methods Phys. Res. Sect. Accel. Spectrometers Detect. Assoc. Equip, Elsevier, 787, pp 98-101,2015 <doi:10.1016/j.nima.2014.11.042>.

[6] J. Krimmer et al., Real-Time Online Monitoring of the Ion Range by Means of Prompt Secondary Radiations, published in 3rd International Conference On Advancements In Nuclear Instrumentation Measurement Methods And Their Applications (ANIMMA 2013), Marseille: France 2013.

[7] S. Deng et al.,Very fast front end ASIC associated with multi-anode PMTs for a scintillating-fibre beam hodoscope, Journal of Instrumentation 8 (2013)C01047. DOI:10.1088/1748-0221/8/01/C01047.

[8] http://e6cvd.com/

[9] http://www.audiatec.de/

[10] M. Schreck et al., Large-area high-quality single crystal diamond, MRS Bulletin, vol.39, nº6, pp. 504-510, 2014.

[11] P. Bergonzo et al., CVD diamond for radiation detection devices, Diamond and Related Materials 10 (2001). 631-638.

[12] E. Berdermann and M. Ciobanu, CVD-Diamond Detectors for Experiments with Hadrons, Nuclei, and Atoms, CVD Diamond for Electronic Devices and Sensors Ed. R. S. Sussmann, 2009 John Wiley \& Sons.

[13] M. Ciobanu et al., In-Beam Diamond Start Detectors, IEEE Trans. Nucl. Sci.58, No. 4 (2011).

[14] F Schirru et al, Development of large area polycrystalline diamond detectors for fast timing application of high-energy heavy-ion beams, JINST No 7 (2012) P05005 doi:10.1088/17480221/7/05/P05005. 
[15] C. Weiss et al., n-TOF collaboration, A new CVD diamond mosaic-detector for $(n, \alpha)$ cross-section measurements at the n-TOF experiment at CERN, proceedings, 13th Vienna Conference on Instrumentation (VCI 2013), Vienna, Austria, February 11-15, 2013, Nucl. Instrum.Meth A732 pp 190194, 2013.

[16] E. Griesmayer and B. Dehning, Diamonds for beam instrumentation, in Proceedings of the 2nd International Conference on Technology and Instrumentation in Particle Physics (TIPP 2011), Chicago U.S.A. (2011) [Phys. Procedia 37 p 1997, 2012].

[17] http://www.ptw.de/2968.html

[18] A. Lacoste et al., Multi-dipolar plasmas for uniform process physics, design and performance, SCi. Technol., 11 pp 407-412, 2002..

[19] https://cividec.at/_C2_Broadband_Amplifier,_2_GHz,_40_dB.html

[20] P. Moritz, E. Berdermann, K. Blasche, H. Stelzer, B. Voss, Broadband electronics for CVD-diamond detectors, Diamond and Related Materials 10 pp 1765-1769, 2001.

[21] D.Breton, E. Delagne, J. Maalmi, Picosecond time measurement using ultra-fast analog memories, proceedings TWEPP2009, Topical Workshop on Electronics for Particle Physics. Paris 21-25 September 2009.

[22] http://teledynelecroy.com/oscilloscope/

[23] M-L Gallin-Martel et al, "Large Area Polycrystalline Diamond Detectors for Online Hadron Therapy Beam Tagging Applications”, IEEE Nuclear Science Symposium \& Medical Imaging Conference (2016 IEEE NSS/MIC), Oct 2016, Strasbourg, France. in press, Proceedings of the IEEE NSS MIC 2016.

[24] M. Kis et al.,’TCT characterization of new generation of DoI samples”, 4th ADAMAS workshop (2014), Dec. 2014, GSI, Darmstadt. 